\title{
An Association of Varicella Zoster Virus, Facial Palsy, and Meningitis in a Young Immunocompetent Male
}

\author{
Amr Ashour Khaldun Obeidat Bahjat Azrieh Arwa Alsaud \\ Mohamed Yassin \\ Department of Medical Education, Hamad Medical Corporation, Doha, Qatar
}

\section{Keywords}

Varicella zoster virus · Shingles · Cerebrospinal fluid · Cranial nerve palsy

\begin{abstract}
Varicella zoster is a secondary infection caused by the virus of chickenpox, after becoming latent in neurons of dorsal root ganglia or trigeminal ganglia. Varicella zoster virus (VZV) can be reactivated years later to produce shingles (zoster), generally in immunocompromised adults to produce neurological deficits and rash. Meningeal involvement of VZV is also reported and can be diagnosed. Herein, we report a 39-year-old immunocompetent male adult with a rare manifestation caused by VZV. The report is an association between facial nerve palsy and a polymerase chain reaction-confirmed VZV meningitis, even without the dermal typical rash. The patient eventually recovered after receiving acyclovir intravenously.
\end{abstract}

(c) 2020 The Author(s)

Published by S. Karger AG, Basel

\section{Introduction}

Varicella, also commonly termed "chickenpox," is an acute and highly contagious disease. It is caused by a primary infection with the varicella zoster virus (VZV). Varicella occurs throughout the world, especially in the absence of a routine vaccination program, and typically affects persons by mid-adulthood. The epidemiology of the disease may relate to the 
properties of VZV (known to be sensitive to heat, climate, population density, and risk of exposure). Following infection, the virus remains latent in nerve cells and may be reactivated causing a secondary infection, herpes zoster, commonly referred to as "shingles." This generally occurs in adults aged over 50 years or in the immunocompromised and is usually associated with a painful rash that may result in permanent spinal nerve damage [1].

VZV may also affect the cranial nerves. Involvement of the trigeminal nerve may cause visual impairment up to blindness (herpes zoster opthalmicus), while involvement of the facial and vestibulocochlear nerves can cause facial paralysis and hearing loss (herpes zoster oticus). These presentations, in particular, require urgent medical attention to prevent serious complications [2].

Infections with neurotropic herpes viruses (such as varicella zoster virus) are frequent in humans. These viruses persist most commonly within cranial nerves, dorsal roots, and autonomic ganglia, causing latent infections with the ability of reactivation [3]. VZV infection of the central nervous system such as encephalitis, meningitis, or myelitis occurs less frequently but is feared because of the numerous unfavorable outcomes [4]. In VZV-associated meningitis, cerebrospinal fluid (CSF) analysis, polymerase chain reaction (PCR), and detection of intrathecal synthesis of antibodies are used to diagnose VZV meningitis [5]. Successful antiviral treatment has been proven by clinical and CSF follow-up examinations [6].

\section{Case Report}

A 39-year-old Bangladeshi gentleman, with no known previous medical illness, presented with a complaint of left-sided facial weakness for 2 days.

Two weeks prior to admission, the patient complained of fever, headache, and sore throat. For the previous complaints, he sought medical advice in the health center. He was given some medications there, saying it was an ulcer, but his symptoms did not improve. After 1 week of the initial symptoms, the patient developed severe left-sided ear pain and tinnitus which was associated with vomiting (3-4 times per day) and dizziness. His symptoms gradually worsened. He then visited a private clinic and was prescribed antibiotics for 5 days. After taking the antibiotics for 3 days, the patient noticed weakness on the left side of his face for which he presented to the emergency department.

Upon admission, the patient was complaining of headache, fever, blurring of vision, ear pain, tinnitus, ear wax discharge, hoarseness of voice, and dysphagia to solids. Detailed physical examination showed facial asymmetry (left 7th nerve upper motor neuron type) and an impairment in the left eye abduction (abducens nerve palsy); other cranial nerves were intact. An important point to note is that there was no rash in his face, throat, or in his ear canal. An urgent head CT scan was done which showed no definite acute intracranial hemorrhage or midline shift or masses. Lumber puncture was safely performed after that. The CSF specimen showed a lymphocytic viral meningitis picture, and VZV PCR was positive.

Head MRI confirmed the presence of left facial nerve neuritis as well, and the patient was started on intravenous acyclovir $10 \mathrm{mg} / \mathrm{kg}$ every $8 \mathrm{~h}$, along with prednisolone for possible Ramsey-Hunt syndrome. He improved gradually and eventually became asymptomatic. 


\section{Discussion}

VZV central nervous system infection can have various presentations, including encephalitis, meningitis, cranial neuropathies, vasculopathy, and myelitis [7]. For example, VZV has been identified as accounting for $15-23 \%$ of viral encephalitis in the USA [8]. However, one of the extraordinary points in this case is the presence of facial palsy (considering clinical presentation and MRI images) in association with confirmed meningitis through detecting VZV chains in the CSF.

Another striking point is that the absence of a rash or history of shingles does not exclude the possibility of VZV meningitis or cranial nerve involvement [9]. This makes identifying VZV as the etiology of central nervous system involvement challenging, especially in the setting of no preceding rash. Nevertheless, a rapid and accurate diagnosis is paramount as early initiation of antivirals is likely to improve patient outcomes [10].

Our case highlights an important phenomenon of whether we can always rely on the hypothesis of the presence of immunocompromising factors in VZV various infections. The abovementioned patient was an immunocompetent healthy adult with no known major illnesses. The literature reports few cases of fulminant disseminated involvement of varicella in young immunocompetent adults who developed hepatitis, severe pneumonia, rhabdomyolysis, and disseminated intravascular coagulation, followed by encephalopathy and multiorgan failure [11].

Early administration of acyclovir-prednisone was proven to reduce nerve degeneration by nerve excitability testing. Hearing recovery also tended to be better in patients with early treatment [12]. A multicenter, randomized, placebo-controlled study showed that valacyclovir and prednisolone therapy was more effective in treating Bell's palsy (excluding zoster sine herpete) than the conventional prednisolone therapy [13].

\section{Conclusion}

In conclusion, we advise physicians to consider VZV infection as a deferential diagnosis in a patient presenting with cranial nerve involvement and symptoms or signs of meningitis. Clinical suspicion of VZV cannot be ruled out, either due to the absence of features of dermal irritation such as rash or in a young and previously healthy immunocompetent patient. We think that the various clinical presentations of VZV cases are underestimated, and detailed CSF diagnostic procedures such as lumber puncture should be considered to detect the virus through PCR or intrathecal synthesis of antiviral antibodies. Treating effectively with valacyclovir and prednisolone is advised as early as possible.

\section{Acknowledgments}

We thank Qatar National Library for funding this article. We would also like to express our appreciation of the Internal Medicine Residency Program of Hamad Medical Corporation. 


\section{Statement of Ethics}

This article was approved by Hamad Medical Corporation Medical Research Center. This research was conducted ethically in accordance with the World Medical Association Declaration of Helsinki. The subject in this paper gave their informed consent to publish their case.

\section{Disclosure Statement}

The authors have no conflicts of interest to disclose.

\section{Funding Sources}

This article was funded by Qatar National Library.

\section{Author Contributions}

Amr Ashour: writing the manuscript. Khaldun Obeidat, Bahjat Azrieh, and Arwa Alsaud: clinical management. Mohamed Yassin: writing and editing.

\section{References}

1 World Health Organization. Immunization, vaccines and biologicals. Varicella. Available from: https://www.who.int/immunization/diseases/varicella/en/.

2 Gilden D, Nagel M, Cohrs R, Mahalingam R, Baird N. Varicella zoster virus in the nervous system. F1000Res. 2015 Nov;4. pii: F1000 Faculty Rev-1356.

3 Gupta P, Ranjan R, Agrawal CS, Muralikrishnan K, Dave N, Rana DS. Meningitis with polymerase chain reaction for varicella zoster positivity in cerebrospinal flid of a young immunocompetent adult. J Neurosci Rural Pract. 2016 Oct-Dec;7(4):591-3.

4 Bienkowski C, Kowalczyk M, Talarek E, Pokorska-Spiewak M, Kierdaszuk B, Marczynska M. Meningitis and Ramsay-Hunt syndrome in a 17-year old girl. Neuro Endocrinol Lett. 2019 Nov;40(3):149-51.

5 Gregoire SM, van Pesch V, Goffette S, Peeters A, Sindic CJ. Polymerase chain reaction analysis and oligoclonal antibody in the cerebrospinal fluid from 34 patients with varicella-zoster virus infection of the nervous system. J Neurol Neurosurg Psychiatry. 2006 Aug;77(8):938-42.

6 Dworkin RH, Johnson RW, Breuer J, Gnann JW, Levin MJ, Backonja M, et al. Recommendations for the management of herpes zoster. Clin Infect Dis. 2007;44(Suppl 1):S1-26.

7 Piquet D. Neurology Midlink. In: Greenlee D, editor. Varicella-zoster virus infections of the nervous system (cited 2019 April 20). University of Colorado, 1977. Available from:

https://www.medlink.com/article/varicella-zoster_virus_infections_of_the_nervous_system.

8 Singh TD, Fugate JE, Rabinstein AA. The spectrum of acute encephalitis: causes, management, and predictors of outcome. Neurology. 2015 Jan;84(4):359-66.

9 Gilden D, Cohrs RJ, Mahalingam R, Nagel MA. Neurological disease produced by varicella zoster virus reactivation without rash. Curr Top Microbiol Immunol. 2010;342:243-53.

10 Erdem H, Cag Y, Ozturk-Engin D, Defres S, Kaya S, Larsen L, et al. Results of a multinational study suggest the need for rapid diagnosis and early antiviral treatment at the onset of herpetic meningoencephalitis. Antimicrob Agents Chemother. 2015;59(6):3084-9.

11 Beby-Defaux A, Brabant S, Chatellier D, Bourgoin A, Robert R, Ruckes T, et al. Disseminated varicella with multiorgan failure in an immunocompetent adult. J Med Virol. 2009 Apr;81(4):747-9.

12 Murakami S, Hato N, Horiuchi J, Honda N, Gyo K, Yanagihara N. Treatment of Ramsay Hunt syndrome with acyclovir-prednisone: significance of early diagnosis and treatment. Ann Neurol. 1997 Mar;41(3):353-7.

13 Hato N, Yamada H, Kohno H, Matsumoto S, Honda N, Gyo K, et al. Valacyclovir and prednisolone treatment for Bell's palsy: a multicenter, randomized, placebo-controlled study. Otol Neurotol. 2007 Apr;28(3):408-13. 